\title{
Crescimento inicial de mudas pré brotadas de cana-de-açúcar submetidas a aplicação de herbicidas ${ }^{1}$
}

\section{Initial growth of sugarcane seedlings submitted to herbicides application}

\author{
Renan dos Santos Sabbag 2 ; Patricia Andrea Monquero ${ }^{3}$; Andreia Cristina Silva Hirata ${ }^{4}$; Paulo \\ Henrique Vieira dos Santos 5
}

\begin{abstract}
Resumo - O método químico de controle de plantas daninhas é o mais utilizado na cultura da cana-de-açúcar, sendo necessários estudos que englobem o efeito destes herbicidas sobre o desenvolvimento de mudas pré-brotadas de cana de açúcar (MPB). O objetivo deste trabalho foi avaliar o efeito da aplicação dos herbicidas ametryn, clomazone, hexazinone + diuron, metribuzin, sulfentrazone e tebuthiuron, isolados e em associação com saflufenacil, no crescimento inicial de mudas pré-brotadas de variedades de cana-de-açúcar. $O$ ensaio foi conduzido em casa de vegetação em delineamento experimental inteiramente casualizado, em esquema fatorial 14 x 2, sendo 14 tratamentos herbicidas e 2 épocas de aplicação após o plantio (3 e 10 DAP), com quatro repetições. Os herbicidas, em suas doses recomendadas, foram aplicados aos 3 ou 10 dias após o transplante das mudas (DAP) das variedades: RB 86 7515, RB 85 5156, RB 966928 e RB 975201 . A porcentagem de fitotoxicidade foi avaliada aos 7, 15, 30 e 60 dias após a aplicação dos tratamentos (DAT). A estatura das plantas, área foliar e biomassa seca da parte aérea foram avaliadas aos 60 DAT. Os herbicidas causaram impacto diferenciado em função das variedades e época de aplicação, todavia, na última avaliação, houve baixa fitotoxicidade na maioria das avaliações. Para a RB 867515 a aplicação dos herbicidas tanto aos 3 como aos 10 DAP foi segura, já para as variedades RB 966928 e RB 975201 a aplicação aos 3 DAP causou menor fitotoxicidade, enquanto, para a RB 855156 foi aos 10 DAP. Conclui-se que as variedades diferem quanto à fitotoxicidade aos herbicidas avaliados, sendo a RB 867515 a mais tolerante e a associação saflufenacil + clomazone e clomazone provocaram maior fitotoxicidade, dentre os tratamentos, nas variedades e épocas estudadas.
\end{abstract}

Palavras-chaves: controle químico; Saccharum officinale; toxicidade

\footnotetext{
Abstract - The chemical weed control is the most used in sugarcane culture, and studies covering the effects of these herbicides on the development of pre-sprouted seedlings of sugarcane. The objective of this work was to evaluate the effect of the herbicides ametryn, clomazone, hexazinone + diuron, metribuzin, sulfentrazone and tebuthiuron isolated and in association with saflufenacil in the initial growth of pre-sprouted seedlings of sugarcane varieties at different times of application.

${ }^{1}$ Recebido para publicação em 08/12/2016 e aceito em 22/02/2017.

2 Aluno do Programa de Mestrado em Agricultura e Ambiente, Centro de Ciências Agrarias/UFSCar. Rodovia Anhanguera, km 174. Araras-SP. E-mail: <renan_sabbag@ @otmail.com>.

3 Professora associada, Departamento de Recursos Naturais e Proteção Ambiental, Centro de Ciências Agrarias/UFSCar. E-mail:< pamonque@cca.ufscar.br>.

${ }^{4}$ Pesquisadora do APTA da Alta Sorocabana, Rodovia Raposo Tavares, km 561, Caixa Postal 298 - CEP: 19015-970, Presidente Prudente-SP. E-mail: <andreiacs@apta.gov.sp.br>.

5 Aluno de graduação do curso de Engenharia Agronômica do Centro de Ciências Agrárias/UFSCar. E-mail: <castelly.sert@hotmail.com>.
} 
The experiment was conducted in a greenhouse in a completely randomized design, in a 14 x 2 factorial scheme, 14 herbicide treatments and 2 application times after planting ( 3 and 10 DAP), with four replications. The herbicides, at their recommended doses, were applied 3 or 10 days after seedling transplant of the varieties: RB 86 7515, RB 85 5156, RB 966928 and RB 975201 . The percentage of phytotoxicity was evaluated at 7, 15, 30 and 60 days after application of the treatments (DAT). The height of the plants, leaf area and dry shoot biomass were evaluated at 60 DAT. The herbicides caused a differential impact according to the varieties and the time of application, however, in the last evaluation, there was low phytotoxicity in most evaluations. For RB 867515 herbicide application at both 3 and 10 DAP was safe, for RB 966928 and RB 975201 the application at 3 DAP caused less phytotoxicity, whereas for RB 855156 it was at 10 DAP. It is concluded that the varieties differ in phytotoxicity to the evaluated herbicides, being RB 867515 the most tolerant and the association saflufenacil + clomazone and clomazone caused greater initial phytotoxicity in the varieties and in the time studied.

Keywords: chemical control; Saccharum officinale; toxicity

\section{Introdução}

O plantio de cana-de-açúcar, por meio de mudas pré-brotadas (MPB), tem se destacado dentre as recentes modificações no setor canavieiro, por ser uma técnica com potencial para substituir o sistema convencional, com economia acima de $20 \mathrm{t} \mathrm{ha}^{-1}$ de mudas de canade-açúcar no plantio. Também ocorre melhor controle de vigor e sanidade, o que permite a redução no volume de mudas, além de sua distribuição espacial viabilizar a irrigação localizada e melhorar o aproveitamento dos nutrientes oriundos da adubação, uma vez que o volume de mudas por metro é menor em ralação ao sistema convencional, havendo redução na competição entre plantas (Landell et al., 2013).

Tanto no plantio de mudas pré-brotadas como no plantio convencional, o controle das plantas daninhas é importante para o pleno desenvolvimento da cultura. Segundo Victoria Filho e Christoffoleti (2004), um dos principais problemas enfrentados pela cultura da cana-deaçúcar é a interferência causada pela presença das plantas daninhas, tanto via competição, alelopatia, como dificultando a colheita, o que pode provocar perdas na produtividade da cultura de até $85 \%$, quando não controladas adequadamente. Existem diferentes métodos de controle das plantas daninhas, sendo o controle químico o mais utilizado em razão de sua alta eficácia, alto rendimento e baixo custo em relação a outros métodos de controle (Pedrinho Jr. et al., 2001).

Dentro deste contexto, existem poucas informações de como os herbicidas se comportarão quando aplicados em área total antes ou logo após o plantio de mudas prébrotadas de cana-de-açúcar, mesmo para os produtos que apresentam seletividade por recomendação em suas respectivas bulas. Em trabalho recente, por exemplo, o herbicida diclosulam, que é seletivo para a cultura da cana-de-açúcar, interferiu negativamente no acúmulo de massa seca da parte aérea e do sistema radicular das variedades CTC 14, CTC 7 e RB 966928 quando aplicado sobre mudas pré-brotadas (Dias, 2014). No mesmo trabalho, os autores não verificaram a mesma resposta com os herbicidas s-metolachlor, clomazone, sulfentrazone, metribuzin, diuron + hexazinone e s metolachor + sulfentrazone.

Em outro trabalho, Rocha Neto (2015) observou que as mudas pré-brotadas da variedade IAC SP95-500 apresentaram apenas leves sintomas de fitotoxicidade (10 a 20\%) e nenhum comprometimento na estatura de plantas com o uso dos herbicidas imazapyr aplicado aos 60 dias antes do plantio; imazapic aplicado aos 45 dias antes do plantio; trifluralin + pendimethalin, diclosulan + s-metolachlor e diclosulan + oxyfluorfen aplicados aos 30 dias antes do plantio; sulfentrazone; amicarbazone e tebuthiuron aplicados aos 2 dias antes do 
plantio, o que possibilita o uso destes produtos no sistema de MPB, sem que ocorra problemas no estabelecimento da planta cultivada.

Estudos tem demonstrado que as variedades de cana-de-açúcar diferem quanto à tolerância aos herbicidas. A variedade RB 855153 , por exemplo, é mais sensível à mistura trifloxysulfuron-sodium + ametryn, quando comparada às variedades RB 835486, RB 845210, RB 867515, RB 928064 (Ferreira et al., 2005). Por outro lado, constataram que a RB867515 se apresentou tolerante às menores doses (1000 e $\left.2000 \mathrm{~g} \mathrm{ha}^{-1}\right)$ do herbicida, porém, na maior dose testada (6000 $\left.\mathrm{g} \mathrm{ha}^{-1}\right)$, o herbicida reduziu o acúmulo de massa seca da parte aérea, a altura, a área foliar e o número de folhas.

A seletividade de herbicidas depende de alguns fatores, tais como as características físico-químicas dos herbicidas, dose, estádio de desenvolvimento da cultura, suscetibilidade dos genótipos e das condições edafoclimáticas no momento da aplicação (Torres et al., 2012). Diversos trabalhos avaliaram a seletividade dos herbicidas tradicionalmente utilizados na canade-açúcar, bem como as diferentes tolerâncias das principais variedades de cana-de-açúcar, porém, são escassas as informações sobre a tolerância de variedades de cana-de-açúcar aos herbicidas quando implantadas por meio de mudas pré-brotadas.

Portanto, o objetivo deste trabalho foi avaliar o efeito de diferentes herbicidas no crescimento inicial de variedades de cana-deaçúcar no sistema de mudas pré-brotadas.

\section{Material e Métodos}

O ensaio foi conduzido em casa de vegetação e as unidades experimentais foram compostas por vasos de polietileno com capacidade volumétrica de 5 litros, preenchidos com solo coletado em local cuja identificação revelou ser do tipo Latossolo Vermelho distrófico. A análise química e física do solo, em amostra coletada na profundidade de $0-20 \mathrm{~cm}$ está apresentada na Tabela 1 .

Tabela 1. Análise química e física do solo utilizado para o preenchimento dos vasos.

\begin{tabular}{ccccccccccccc}
\hline $\mathrm{pH}$ & $\mathrm{MO}$ & $\mathrm{P}$ & $\mathrm{K}^{+}$ & $\mathrm{Ca}^{+2}$ & $\mathrm{Mg}^{+2}$ & $\mathrm{H}^{+}+\mathrm{Al}^{+3}$ & $\mathrm{SB}$ & $\mathrm{CTC}$ & $\mathrm{V}$ & Argila & Silte & Areia \\
\hline $\mathrm{CaCl}_{2}$ & $\mathrm{~g} \mathrm{dm}^{-3}$ & $\mathrm{mg} \mathrm{dm}^{-3}$ & & & & $\mathrm{mmolc} \mathrm{dm}^{-3}$ & & & $\%$ & & $\mathrm{~g} \mathrm{~kg}^{-1}$ & \\
\hline 5,7 & 22 & 16 & 2,8 & 37 & 14 & 22 & 53,8 & 75,8 & 71 & 560 & 240 & 200 \\
\hline
\end{tabular}

Quatro variedades de cana-de-açúcar (RB 86 7515, RB 85 5156, RB 966928 e RB 97 5201) foram plantadas utilizando-se mudas prébrotadas (MPBs), sendo que a tecnologia adotada foi o de agricultura de mudas sadias (AgMusa), da empresa BASF. As mudas foram plantadas 60 dias após a brotação das gemas a irrigação foi realizada por sistema de aspersão.

As variedades foram avaliadas em experimentos separados, todos realizados em delineamento inteiramente casualizado, no esquema fatorial 14 × 2 , sendo o primeiro fator constituídos dos herbicidas aplicados (Tabela 2) e, o segundo, das 2 épocas de aplicação após o plantio (3 e 10 DAP), com quatro repetições.

$\mathrm{O}$ equipamento utilizado para aplicação dos herbicidas foi o pulverizador costal pressurizado de ar comprimido, com barra de aplicação de $3 \mathrm{~m}$, espaçamento entre bicos de $0,5 \mathrm{~m}$, sendo os mesmos do tipo leque, modelo XR 110.02. A vazão utilizada foi de $150 \mathrm{~L} \mathrm{ha}^{-1}$, sendo a temperatura do ar de $24^{\circ} \mathrm{C}$, umidade relativa do ar de $57 \%$ e velocidade do vento de 4 a $6 \mathrm{~km} \mathrm{~h}^{-1}$.

A fitotoxicidade dos herbicidas nas mudas de cana-de-açúcar foi avaliada aos 7, 15, 30 e 60 dias após a aplicação dos tratamentos (DAT), utilizando-se a escala de notas da ALAM (1974), onde 0\% significa nenhuma injúria e $100 \%$ a morte total das plantas.

Aos 60 DAT foi determinada a estatura $(\mathrm{cm})$, a partir da base até a inserção da primeira folha, a área foliar $\left(\mathrm{cm}^{2}\right)$, utilizando um medidor de área foliar portátil (LICOR 3000C) e biomassa seca da parte aérea $(\mathrm{g})$, sendo para isto 
as mudas cortadas rente ao solo e colocadas em estufa de circulação forçada de ar a $65^{\circ} \mathrm{C}$ por 48 horas, e pesadas posteriormente em balança analítica.

Tabela 2. Herbicidas aplicados aos 3 ou 10 DAP de mudas pré-brotadas de cana de açúcar.

\begin{tabular}{lccc}
\hline \multicolumn{1}{c}{ Herbicida } & Produto comercial & Dose $\left(\mathrm{g} \mathrm{i.a.} \mathrm{ha} \mathrm{a}^{-1}\right)$ & Dose (kg ou L p.c. ha $\left.\mathrm{f}^{-1}\right)$ \\
\hline Saflufenacil & Heat & 98 & 0,140 \\
Ametryn & Gesapax & 3000 & 6,0 \\
Sulfentrazone & Boral & 700 & 1,4 \\
Tebuthiuron & Combine & 800 & 1,6 \\
[Diuron + hexazinone] & Velpar K & {$[1170+330]$} & 2,5 \\
Metribuzin & Sencor & 1680 & 3,5 \\
Clomazone & Gamit Star & 1120 & 1,4 \\
Saflufenacil + ametryn & Heat + Gesapax & $98+3000$ & $0,140+6,0$ \\
Saflufenacil + sulfentrazone & Heat + Boral & $98+700$ & $0,140+1,4$ \\
Saflufenacil + tebuthiuron & Heat + Combine & $98+800$ & $0,140+1,6$ \\
Saflufenacil + [diuron + hexazinone] & Heat + Velpar K & $98+[1170+330]$ & $0,140+2,5$ \\
Saflufenacil + metribuzin & Heat + Sencor & $98+1680$ & $0,140+3,5$ \\
Saflufenacil + clomazone & Heat + Gamit & $98+1120$ & $0,140+1,4$ \\
Testemunha & & & \\
\hline
\end{tabular}

Os dados obtidos foram submetidos à análise de variância, e quando significativas, as médias comparadas pelo teste de Scott-Knott a $5 \%$ de probabilidade.

\section{Resultados e Discussão}

Não foram detectadas diferenças estatísticas entre os tratamentos herbicidas e a testemunha na aplicação aos 3 DAP para a variedade RB 867515 na primeira avaliação aos 7 DAT (Tabela 3). Aos 15 DAT, a associação saflufenacil + clomazone provocou fitotoxicidade de $77 \%$, o que pode comprometer o crescimento inicial das plantas e o fechamento na entrelinha. Os tratamentos envolvendo os herbicidas hexazinone + diuron $(5 \%)$, metribuzin $(1 \%)$, saflufenacil + diuron + hexazinone $(13 \%)$, saflufenacil + metribuzin (12\%), saflufenacil + ametryn (19\%) e clomazone (19\%) não diferiram da testemunha.

A fitotoxicidade aos 30 DAT, no geral, foi reduzida, porém a associação saflufenacil + clomazone e o tratamento com tebuthiuron causaram fitotoxicidade de 50 e 39\%, respectivamente (Tabela 3). Aos 60 DAA os tratamentos que provocaram maior fitotoxicidade nas mudas foram: tebuthiuron
$(36 \%)$, saflufenacil + ametryn $(39 \%)$ e saflufenacil + clomazone $(56 \%)$.

Ao longo das avaliações, observou-se que a associação saflufenacil + clomazone apresentou maior fitotoxicidade na avaliação realizada aos 15 DAA, com folhas albinas e necrose pontual, com posterior recuperação, já os demais tratamentos foram estáveis (Tabela $3)$.

Na aplicação aos 10 DAP, observa-se que aos 7 DAA a associação saflufenacil + clomazone provocou 50\% de fitotoxicidade nas mudas, seguido por saflufenacil + sulfentrazone com 32\% de fitotoxicidade (Tabela 3). Na avaliação realizada aos 15 DAA, o único tratamento que apresentou diferença com relação à testemunha foi a associação saflufenacil + clomazone, com $62 \%$ de fitotoxicidade. $O$ mesmo padrão pode ser observado nas avaliações de 30 e 60 DAA (Tabela 3). Desse modo, pode ser verificado que a associação de clomazone com saflufenacil tem restrição quanto ao uso sobre as mudas prébrotadas desta variedade, diferentemente dos mesmos herbicidas aplicados isoladamente. $\mathrm{O}$ potencial destas associações de provocar maior fitotoxicidade já tinha sido observado por Inoue et al. (2007) que relataram que alguns herbicidas inibidores de fotossíntese (FS II), como, por 
exemplo, hexazinone + diuron e ametryn tornam-se mais fitotóxicos para a cana-deaçúcar quando usados em associação com outros herbicidas inibidores de pigmentos (carotenos), como é o caso do clomazone.

Tabela 3. Porcentagem de fitotoxicidade dos herbicidas aplicados nas mudas pré-brotadas, na variedade RB 867515 , após 3 e 10 DAP.

\begin{tabular}{|c|c|c|c|c|c|c|c|c|}
\hline \multirow{2}{*}{ Tratamentos } & \multicolumn{4}{|c|}{3 DAP } & \multicolumn{4}{|c|}{$10 \mathrm{DAP}$} \\
\hline & 7 DAA & $15 \mathrm{DAA}$ & 30 DAA & 60 DAA & 7 DAA & $15 \mathrm{DAA}$ & 30 DAA & 60 DAA \\
\hline SAF & $15 \mathrm{aA}$ & $26 \mathrm{~A}$ & $16 \mathrm{bA}$ & $12 \mathrm{bA}$ & $14 \mathrm{cA}$ & $10 \mathrm{bA}$ & $10 \mathrm{bA}$ & $14 \mathrm{bA}$ \\
\hline AME & $22 \mathrm{aA}$ & $34 \mathrm{bA}$ & $26 \mathrm{aA}$ & $31 \mathrm{aA}$ & $3 \mathrm{cA}$ & $11 \mathrm{bA}$ & $9 \mathrm{bA}$ & $11 \mathrm{bA}$ \\
\hline SUL & $24 \mathrm{aA}$ & $30 \mathrm{bA}$ & $16 \mathrm{bA}$ & $16 \mathrm{bA}$ & $22 \mathrm{cA}$ & $20 \mathrm{bA}$ & $17 \mathrm{bA}$ & $22 \mathrm{bA}$ \\
\hline TEB & $19 \mathrm{aA}$ & $36 \mathrm{bA}$ & $39 \mathrm{aA}$ & $36 \mathrm{aA}$ & $10 \mathrm{cA}$ & $12 \mathrm{bA}$ & $3 \mathrm{bA}$ & $9 \mathrm{bA}$ \\
\hline$[\mathrm{DIU}+\mathrm{HEX}]$ & $3 \mathrm{aA}$ & $5 \mathrm{cA}$ & $0 \mathrm{bA}$ & $7 \mathrm{bA}$ & $5 \mathrm{cA}$ & $12 \mathrm{bA}$ & $11 \mathrm{bA}$ & $15 \mathrm{bA}$ \\
\hline MET & 3 aA & $1 \mathrm{cA}$ & $0 \mathrm{bA}$ & $2 \mathrm{bA}$ & $4 \mathrm{cA}$ & $4 \mathrm{bA}$ & $2 \mathrm{bA}$ & $8 \mathrm{bA}$ \\
\hline CLO & $7 \mathrm{aA}$ & $19 \mathrm{cA}$ & $17 \mathrm{bA}$ & $22 \mathrm{bA}$ & $12 \mathrm{cA}$ & $16 \mathrm{bA}$ & $15 \mathrm{bA}$ & $20 \mathrm{bA}$ \\
\hline $\mathrm{SAF}+\mathrm{AME}$ & $19 \mathrm{aA}$ & $19 \mathrm{cA}$ & $16 \mathrm{bA}$ & $39 \mathrm{aA}$ & $16 \mathrm{cA}$ & $12 \mathrm{bA}$ & $11 \mathrm{bA}$ & $15 \mathrm{bA}$ \\
\hline SAF + SUL & $29 \mathrm{aA}$ & $42 \mathrm{bA}$ & $19 \mathrm{bA}$ & $17 \mathrm{bA}$ & $32 \mathrm{bA}$ & $19 \mathrm{bB}$ & $12 \mathrm{bB}$ & $11 \mathrm{bB}$ \\
\hline $\mathrm{SAF}+\mathrm{TEB}$ & $12 \mathrm{aA}$ & $27 \mathrm{bA}$ & $15 \mathrm{bA}$ & $14 \mathrm{bA}$ & $16 \mathrm{cA}$ & $14 \mathrm{bA}$ & $11 \mathrm{bA}$ & $24 \mathrm{bA}$ \\
\hline $\mathrm{SAF}+[\mathrm{DIU}+\mathrm{HEX}]$ & $10 \mathrm{aA}$ & $13 \mathrm{cA}$ & $12 \mathrm{bA}$ & $11 \mathrm{bA}$ & $17 \mathrm{cA}$ & $22 \mathrm{bA}$ & $22 \mathrm{bA}$ & $24 \mathrm{bA}$ \\
\hline SAF + MET & 9 aA & $12 \mathrm{cA}$ & $9 \mathrm{bA}$ & $21 \mathrm{bA}$ & $17 \mathrm{cA}$ & $15 \mathrm{bA}$ & $12 \mathrm{bA}$ & $17 \mathrm{bA}$ \\
\hline $\mathrm{SAF}+\mathrm{CLO}$ & $42 \mathrm{aB}$ & $77 \mathrm{aA}$ & $50 \mathrm{aB}$ & $56 \mathrm{aB}$ & $50 \mathrm{aA}$ & $62 \mathrm{aA}$ & $57 \mathrm{aA}$ & $54 \mathrm{aA}$ \\
\hline Testemunha & $0 \mathrm{aA}$ & $0 \mathrm{cA}$ & $0 \mathrm{bA}$ & $0 \mathrm{bA}$ & $0 \mathrm{cA}$ & $0 \mathrm{bA}$ & $0 \mathrm{bA}$ & $0 \mathrm{bA}$ \\
\hline $\mathrm{CV}(\%)$ & \multicolumn{4}{|c|}{98} & \\
\hline
\end{tabular}

$\overline{\mathrm{SAF}}=$ saflufenacil; $\mathrm{AME}=$ ametryn$; \mathrm{SUL}=$ sulfentrazone; TEB $=$ tebuthiuron; HEX $=$ hexazinone; $\mathrm{DIU}=$ diuron; MET = metribuzin; $\mathrm{CLO}=$ clomazone. Médias seguidas por letras minúsculas iguais na coluna e maiúsculas na linha não diferem entre si pelo teste de Scott-Knott ao nível de 5\% de probabilidade.

Em trabalho de Costa et al. (2012), foi observado que o saflufenacil, associado a mistura pronta de ametryn + clomazone, também causou fitotoxicidade acima de 30\%, aos 15 dias após sua aplicação nas variedades SP $80-1816$ e SP $81-3250$ e $24 \%$ na variedade RB 855156. Vale ressaltar que neste caso os herbicidas foram aplicados sobre plantas provenientes de tolete, talvez, por isto, a fitotoxicidade foi menor do que a observada no presente trabalho.

As avaliações biométricas (estatura, área foliar e biomassa) na variedade RB 867515 , evidenciam que não houve diferença estatística nestas variáveis quando a aplicação dos tratamentos ocorreu aos 3 DAP, com exceção da ametryn cuja planta apresentou área foliar maior que os demais tratamentos (Tabela 4). Quando os herbicidas foram aplicados aos 10 DAP, o único parâmetro que apresentou diferenças estatísticas foi a estatura, porém se observa que a testemunha apresentou menor estatura em relação a outros tratamentos, o que evidencia que essa diferença não teve relação com a presença dos herbicidas (Tabela 4). Estes dados corroboram com Barela e Christoffoleti (2006) que já tinham observado a tolerância da variedade RB 867515 a diferentes herbicidas como tebuthiuron, sulfentrazone, metribuzin, diuron + hexazinone, clomazone e ametryn, porém, no trabalho destes autores a aplicação foi em pré-emergência da cultura.

A variedade RB 85 5156, apresentou tolerância a vários dos tratamentos aplicados aos 3 DAP, sendo que as maiores fitotoxicidade, aos 7 DAA, foram observadas com o uso da associação saflufenacil + clomazone (22\%) e saflufenacil + sulfentrazone $(20 \%)$ (Tabela 5).

$\mathrm{Na}$ avaliação realizada aos 15 DAA, destacam-se de maneira negativa, as associações de saflufenacil + clomazone (34\%), sulfentrazone $(29 \%)$, ametryn $(26 \%)$ e o uso de clomazone isolado $(27 \%)$. 
Tabela 4. Estatura (cm), Área foliar $\left(\mathrm{cm}^{2}\right)$ e Biomassa (g) das mudas pré-brotadas, na variedade RB 86 7515, submetidas a diferentes herbicidas aos 3 e 10 DAP.

\begin{tabular}{lcccccc}
\hline \multirow{2}{*}{ Tratamentos } & \multicolumn{3}{c}{3 DAP } & \multicolumn{1}{c}{ DAP } \\
\cline { 2 - 7 } & $\begin{array}{c}\text { Estatura } \\
(\mathrm{cm})\end{array}$ & $\begin{array}{c}\text { Área Foliar } \\
\left(\mathrm{cm}^{2}\right)\end{array}$ & $\begin{array}{c}\text { Biomassa } \\
(\mathrm{g})\end{array}$ & $\begin{array}{c}\text { Estatura } \\
(\mathrm{cm})\end{array}$ & $\begin{array}{c}\text { Área Foliar } \\
\left(\mathrm{cm}^{2}\right)\end{array}$ & $\begin{array}{c}\text { Biomassa } \\
(\mathrm{g})\end{array}$ \\
\hline SAF & $8,95 \mathrm{a}$ & $44,23 \mathrm{a}$ & $0,32 \mathrm{a}$ & $9,00 \mathrm{a}$ & $55,41 \mathrm{a}$ & $0,26 \mathrm{a}$ \\
AME & $10,20 \mathrm{a}$ & $108,03 \mathrm{~b}$ & $0,62 \mathrm{a}$ & $8,82 \mathrm{a}$ & $54,63 \mathrm{a}$ & $0,25 \mathrm{a}$ \\
SUL & $7,35 \mathrm{a}$ & $70,06 \mathrm{a}$ & $0,34 \mathrm{a}$ & $7,00 \mathrm{~b}$ & $40,21 \mathrm{a}$ & $0,28 \mathrm{a}$ \\
TEB & $7,45 \mathrm{a}$ & $75,12 \mathrm{a}$ & $0,26 \mathrm{a}$ & $10,02 \mathrm{a}$ & $62,35 \mathrm{a}$ & $0,28 \mathrm{a}$ \\
[DIU + HEX] & $7,55 \mathrm{a}$ & $61,29 \mathrm{a}$ & $0,49 \mathrm{a}$ & $7,80 \mathrm{~b}$ & $50,84 \mathrm{a}$ & $0,24 \mathrm{a}$ \\
MET & $9,00 \mathrm{a}$ & $68,06 \mathrm{a}$ & $0,56 \mathrm{a}$ & $8,80 \mathrm{a}$ & $47,67 \mathrm{a}$ & $0,37 \mathrm{a}$ \\
CLO & $7,00 \mathrm{a}$ & $52,62 \mathrm{a}$ & $0,39 \mathrm{a}$ & $8,00 \mathrm{~b}$ & $44,99 \mathrm{a}$ & $0,28 \mathrm{a}$ \\
SAF+ AME & $5,80 \mathrm{a}$ & $70,52 \mathrm{a}$ & $0,49 \mathrm{a}$ & $8,92 \mathrm{a}$ & $58,96 \mathrm{a}$ & $0,25 \mathrm{a}$ \\
SAF+ SUL & $7,55 \mathrm{a}$ & $47,17 \mathrm{a}$ & $0,26 \mathrm{a}$ & $9,87 \mathrm{a}$ & $67,36 \mathrm{a}$ & $0,29 \mathrm{a}$ \\
SAF+ TEB & $8,35 \mathrm{a}$ & $79,13 \mathrm{a}$ & $0,41 \mathrm{a}$ & $9,00 \mathrm{a}$ & $55,93 \mathrm{a}$ & $0,32 \mathrm{a}$ \\
SAF + [DIU + HEX] & $7,10 \mathrm{a}$ & $51,56 \mathrm{a}$ & $0,36 \mathrm{a}$ & $8,12 \mathrm{~b}$ & $41,21 \mathrm{a}$ & $0,22 \mathrm{a}$ \\
SAF+ MET & $8,05 \mathrm{a}$ & $62,13 \mathrm{a}$ & $0,44 \mathrm{a}$ & $9,05 \mathrm{a}$ & $46,97 \mathrm{a}$ & $0,42 \mathrm{a}$ \\
SAF + CLO & $6,05 \mathrm{a}$ & $47,42 \mathrm{a}$ & $0,14 \mathrm{a}$ & $7,37 \mathrm{~b}$ & $60,67 \mathrm{a}$ & $0,16 \mathrm{a}$ \\
Testemunha & $7,80 \mathrm{a}$ & $51,79 \mathrm{a}$ & $0,44 \mathrm{a}$ & $7,80 \mathrm{~b}$ & $51,79 \mathrm{a}$ & $0,44 \mathrm{a}$ \\
\hline CV $(\%)$ & 27,78 & 34,28 & 61,71 & 16,17 & 27,33 & 72,04 \\
\hline
\end{tabular}

SAF = saflufenacil; AME = ametryn; SUL = sulfentrazone; TEB = tebuthiuron; HEX = hexazinone; DIU = diuron; MET = metribuzin; $\mathrm{CLO}=$ clomazone. Médias seguidas por letras minúsculas iguais na coluna e maiúsculas na linha não diferem entre si pelo teste de Scott-Knott ao nível de 5\% de probabilidade.

Tabela 5. Porcentagem de fitotoxicidade dos herbicidas aplicados nas mudas pré-brotadas, na variedade RB 85 5156, após 3 e 10 DAP.

\begin{tabular}{lcccccccc}
\hline \multirow{2}{*}{ Tratamentos } & \multicolumn{4}{c}{$3 \mathrm{DAP}$} & \multicolumn{4}{c}{$10 \mathrm{DAP}$} \\
\cline { 2 - 8 } & $7 \mathrm{DAA}$ & $15 \mathrm{DAA}$ & $30 \mathrm{DAA}$ & $60 \mathrm{DAA}$ & $7 \mathrm{DAA}$ & $15 \mathrm{DAA}$ & $30 \mathrm{DAA}$ & $60 \mathrm{DAA}$ \\
\hline SAF & $11 \mathrm{bA}$ & $11 \mathrm{cA}$ & $10 \mathrm{cA}$ & $9 \mathrm{eA}$ & $12 \mathrm{bA}$ & $7 \mathrm{cA}$ & $12 \mathrm{bA}$ & $9 \mathrm{cA}$ \\
AME & $5 \mathrm{cB}$ & $21 \mathrm{bA}$ & $9 \mathrm{cB}$ & $4 \mathrm{eB}$ & $7 \mathrm{cA}$ & $6 \mathrm{dA}$ & $10 \mathrm{bA}$ & $7 \mathrm{cA}$ \\
SUL & $12 \mathrm{bA}$ & $14 \mathrm{cA}$ & $12 \mathrm{cA}$ & $20 \mathrm{cA}$ & $12 \mathrm{bA}$ & $11 \mathrm{cA}$ & $14 \mathrm{bA}$ & $15 \mathrm{bA}$ \\
TEB & $5 \mathrm{cA}$ & $4 \mathrm{dA}$ & $2 \mathrm{dA}$ & $2 \mathrm{eA}$ & $15 \mathrm{bA}$ & $11 \mathrm{cA}$ & $6 \mathrm{cB}$ & $7 \mathrm{cB}$ \\
{$[\mathrm{DIU}+\mathrm{HEX}]$} & $6 \mathrm{cB}$ & $14 \mathrm{cB}$ & $24 \mathrm{bA}$ & $22 \mathrm{cA}$ & $4 \mathrm{cC}$ & $10 \mathrm{cC}$ & $19 \mathrm{aB}$ & $30 \mathrm{aA}$ \\
MET & $2 \mathrm{cA}$ & $9 \mathrm{cA}$ & $0 \mathrm{dA}$ & $0 \mathrm{eA}$ & $5 \mathrm{cA}$ & $3 \mathrm{dA}$ & $8 \mathrm{bA}$ & $6 \mathrm{cA}$ \\
CLO & $12 \mathrm{bC}$ & $27 \mathrm{aB}$ & $32 \mathrm{aB}$ & $54 \mathrm{aA}$ & $15 \mathrm{bB}$ & $19 \mathrm{bB}$ & $25 \mathrm{aA}$ & $25 \mathrm{aA}$ \\
SAF + AME & $10 \mathrm{bB}$ & $26 \mathrm{aA}$ & $20 \mathrm{bA}$ & $12 \mathrm{~dB}$ & $12 \mathrm{bA}$ & $12 \mathrm{cA}$ & $11 \mathrm{bA}$ & $14 \mathrm{cA}$ \\
SAF + SUL & $20 \mathrm{aA}$ & $29 \mathrm{aA}$ & $21 \mathrm{bA}$ & $21 \mathrm{cA}$ & $19 \mathrm{aA}$ & $17 \mathrm{bA}$ & $16 \mathrm{aA}$ & $20 \mathrm{bA}$ \\
SAF + TEB & $15 \mathrm{bA}$ & $19 \mathrm{bA}$ & $12 \mathrm{cA}$ & $12 \mathrm{dA}$ & $11 \mathrm{bA}$ & $10 \mathrm{cA}$ & $12 \mathrm{bA}$ & $9 \mathrm{cA}$ \\
SAF + [DIU + HEX] & $7 \mathrm{cB}$ & $12 \mathrm{cB}$ & $16 \mathrm{bA}$ & $19 \mathrm{cA}$ & $14 \mathrm{bA}$ & $19 \mathrm{bA}$ & $20 \mathrm{aA}$ & $21 \mathrm{bA}$ \\
SAF + MET & $11 \mathrm{bB}$ & $16 \mathrm{cA}$ & $17 \mathrm{bA}$ & $7 \mathrm{eB}$ & $14 \mathrm{bA}$ & $15 \mathrm{bA}$ & $14 \mathrm{bA}$ & $19 \mathrm{bA}$ \\
SAF + CLO & $22 \mathrm{aB}$ & $34 \mathrm{aA}$ & $27 \mathrm{aB}$ & $39 \mathrm{bA}$ & $21 \mathrm{aA}$ & $27 \mathrm{aA}$ & $22 \mathrm{aA}$ & $20 \mathrm{bA}$ \\
Testemunha & $0 \mathrm{cA}$ & $0, \mathrm{dA}$ & $0, \mathrm{dA}$ & $0 \mathrm{eA}$ & $0 \mathrm{cA}$ & $0 \mathrm{dA}$ & $0 \mathrm{cA}$ & $0 \mathrm{dA}$ \\
\hline CV\% & \multicolumn{3}{c}{39} & & & & & \\
\hline
\end{tabular}

$\overline{\mathrm{SAF}}=$ saflufenacil; $\mathrm{AME}=$ ametryn$; \mathrm{SUL}=$ sulfentrazone; $\mathrm{TEB}=$ tebuthiuron; HEX $=$ hexazinone; $\mathrm{DIU}=$ diuron; $\mathrm{MET}=$ metribuzin ; $\mathrm{CLO}=$ clomazone. Médias seguidas por letras minúsculas iguais na coluna e maiúsculas na linha não diferem entre si pelo teste de Scott-Knott ao nível de 5\% de probabilidade.

Aos 30 e 60 DAA, observa-se que, em alguns tratamentos, houve recuperação das plantas, sendo que clomazone e saflufenacil + clomazone apresentaram maior fitotoxicidade. $\mathrm{Na}$ aplicação aos 10 DAP não foi verificada fitotoxicidade maior que $30 \%$, entretanto, destacam-se na última avaliação, os herbicidas clomazone e diuron + hexazinone com 25 e $30 \%$ de fitotoxicidade, respectivamente. Ao longo das avaliações, excetuando-se os tratamentos diuron + hexazinone e clomazone não se observou aumento da fitotoxicidade (Tabela 5). 
Os dados biométricos para a aplicação realizada aos 3 DAP evidenciam que não houve efeito dos tratamentos na estatura e na área foliar das plantas, porém a biomassa seca da parte aérea foi influenciada negativamente pelos herbicidas, à exceção do saflufenacil, ametryn, metribuzin e saflufenacil + clomazone (Tabela
6). Quando os herbicidas foram aplicados sobre as mudas aos 10 DAP, não se verificou diferenças estatísticas em nenhum dos parâmetros biométricos avaliados, ou seja, a fitotoxicidade provocada por alguns tratamentos, não afetou o crescimento das plantas (Tabela 6).

Tabela 6. Estatura (cm), área foliar $\left(\mathrm{cm}^{2}\right)$ e biomassa $(\mathrm{g})$ das mudas pré-brotadas, na variedade RB 855156 , submetidas a diferentes herbicidas aos 3 e 10 DAP.

\begin{tabular}{lcccccc}
\hline \multirow{2}{*}{ Tratamentos } & \multicolumn{3}{c}{3 DAP } & \multicolumn{3}{c}{$10 \mathrm{DAP}$} \\
\cline { 2 - 6 } & Estatura & Área foliar & Biomassa & Estatura & Área foliar & Biomassa \\
\hline SAF & $7,37 \mathrm{a}$ & $60,94 \mathrm{a}$ & $1,89 \mathrm{a}$ & $7,25 \mathrm{a}$ & $53,97 \mathrm{a}$ & $1,27 \mathrm{a}$ \\
AME & $7,80 \mathrm{a}$ & $34,81 \mathrm{a}$ & $1,54 \mathrm{a}$ & $7,87 \mathrm{a}$ & $63,29 \mathrm{a}$ & $1,16 \mathrm{a}$ \\
SUL & $7,00 \mathrm{a}$ & $51,56 \mathrm{a}$ & $0,86 \mathrm{~b}$ & $8,02 \mathrm{a}$ & $46,79 \mathrm{a}$ & $0,76 \mathrm{a}$ \\
TEB & $7,87 \mathrm{a}$ & $47,66 \mathrm{a}$ & $0,68 \mathrm{~b}$ & $7,30 \mathrm{a}$ & $50,48 \mathrm{a}$ & $0,73 \mathrm{a}$ \\
[DIU + HEX] & $7,00 \mathrm{a}$ & $40,86 \mathrm{a}$ & $1,09 \mathrm{~b}$ & $6,80 \mathrm{a}$ & $31,60 \mathrm{a}$ & $0,71 \mathrm{a}$ \\
MET & $9,25 \mathrm{a}$ & $56,26 \mathrm{a}$ & $2,01 \mathrm{a}$ & $6,75 \mathrm{a}$ & $46,89 \mathrm{a}$ & $1,32 \mathrm{a}$ \\
CLO & $4,10 \mathrm{a}$ & $27,41 \mathrm{a}$ & $0,79 \mathrm{~b}$ & $5,87 \mathrm{a}$ & $48,58 \mathrm{a}$ & $0,60 \mathrm{a}$ \\
SAF + AME & $6,35 \mathrm{a}$ & $40,66 \mathrm{a}$ & $0,94 \mathrm{~b}$ & $6,50 \mathrm{a}$ & $40,17 \mathrm{a}$ & $0,80 \mathrm{a}$ \\
SAF + SUL & $6,75 \mathrm{a}$ & $48,87 \mathrm{a}$ & $0,61 \mathrm{~b}$ & $7,25 \mathrm{a}$ & $41,41 \mathrm{a}$ & $0,63 \mathrm{a}$ \\
SAF + TEB & $6,42 \mathrm{a}$ & $41,09 \mathrm{a}$ & $1,09 \mathrm{~b}$ & $8,17 \mathrm{a}$ & $60,42 \mathrm{a}$ & $0,72 \mathrm{a}$ \\
SAF + [DIU + HEX] & $9,50 \mathrm{a}$ & $39,17 \mathrm{a}$ & $1,09 \mathrm{~b}$ & $6,85 \mathrm{a}$ & $40,76 \mathrm{a}$ & $0,59 \mathrm{a}$ \\
SAF + MET & $7,82 \mathrm{a}$ & $33,53 \mathrm{a}$ & $1,13 \mathrm{~b}$ & $7,00 \mathrm{a}$ & $35,36 \mathrm{a}$ & $0,82 \mathrm{a}$ \\
SAF + CLO & $6,50 \mathrm{a}$ & $44,43 \mathrm{a}$ & $1,47 \mathrm{a}$ & $7,57 \mathrm{a}$ & $52,78 \mathrm{a}$ & $0,90 \mathrm{a}$ \\
Testemunha & $7,57 \mathrm{a}$ & $52,17 \mathrm{a}$ & $1,39 \mathrm{a}$ & $7,57 \mathrm{a}$ & $52,17 \mathrm{a}$ & $1,39 \mathrm{a}$ \\
\hline CV $(\%)$ & 21,78 & 37,07 & 51,72 & 16,61 & 29,34 & 76,65 \\
\hline
\end{tabular}

SAF = saflufenacil; AME = ametryn; SUL = sulfentrazone; TEB = tebuthiuron; HEX = hexazinone; DIU = diuron; MET = metribuzin; $\mathrm{CLO}=$ clomazone. Médias seguidas por letras minúsculas iguais na coluna e maiúsculas na linha não diferem entre si pelo teste de Scott-Knott ao nível de 5\% de probabilidade.

A questão de se observar fitotoxicidade, mas não verificar influência em parâmetros de crescimento/desenvolvimento, foi visto também por Martins et al. (2005) que trabalhando com cana-de-açúcar proveniente de tolete, observaram que embora tenham sido verificados sintomas de fitotoxicidade nas plantas pelo uso do herbicida diclosulam, a variedade RB 85 5156, não diferiu estatisticamente da testemunha em relação a variável altura de colmo.

A fitotoxicidade para a variedade RB 96 6928 foi baixa para a maioria dos tratamentos tanto aos 3 como aos 10 DAP. Os resultados relativos à aplicação aos 3 DAP mostraram que, na última avaliação, apenas o herbicida clomazone apresentou maior fitotoxicidade (22\%) (Tabela 7). Houve tendência de redução da fitotoxicidade no decorrer das avaliações para a maioria dos herbicidas.

$\mathrm{Na}$ aplicação realizada aos $10 \mathrm{DAP}$, os maiores valores de fitotoxicidade aos 7 DAA foram encontrados nos tratamentos com saflufenacil (24\%), sulfentrazone (29\%), clomazone $(24 \%)$, saflufenacil + sulfentrazone (19\%) e saflufenacil + clomazone (21\%). Já as 60 DAA as maiores fitotoxicidade foram provocadas pelos tratamentos saflufenacil + [diuron + hexazinone] (21\%), clomazone (20\%) e saflufenacil + ametryn (17\%) (Tabela 7).

Na variedade RB 96 6928, houve efeito negativo dos tratamentos na estatura das plantas, todavia, a área foliar foi igual ou até mesmo maior em alguns tratamentos em relação à testemunha, sendo que a biomassa não diferiu na aplicação realizada aos 3 DAP (Tabela 8). 
Tabela 7. Porcentagem de fitotoxicidade dos herbicidas aplicados nas mudas pré-brotadas, na variedade RB 96 6928, após 3 e 10 DAP.

\begin{tabular}{|c|c|c|c|c|c|c|c|c|}
\hline \multirow{2}{*}{ Tratamentos } & \multicolumn{4}{|c|}{3 DAP } & \multicolumn{4}{|c|}{10 DAP } \\
\hline & 7 DAA & 15 DAA & 30 DAA & 60 DAA & 7 DAA & $15 \mathrm{DAA}$ & 30 DAA & 60 DAA \\
\hline SAF & $17 \mathrm{aA}$ & $17 \mathrm{cA}$ & $10 \mathrm{cB}$ & $9 \mathrm{cB}$ & $24 \mathrm{aA}$ & $19 \mathrm{bA}$ & $17 \mathrm{aA}$ & $12 \mathrm{bA}$ \\
\hline AME & $10 \mathrm{cB}$ & $17 \mathrm{cA}$ & $14 \mathrm{bA}$ & $7 \mathrm{cB}$ & $12 \mathrm{bA}$ & $7 \mathrm{cA}$ & $7 \mathrm{bA}$ & $7 \mathrm{cA}$ \\
\hline SUL & $15 \mathrm{bA}$ & $15 \mathrm{cA}$ & $14 \mathrm{bA}$ & $9 \mathrm{cB}$ & $29 \mathrm{aA}$ & $26 \mathrm{aA}$ & $15 \mathrm{aB}$ & $14 \mathrm{bB}$ \\
\hline TEB & $9 \mathrm{cA}$ & $10 \mathrm{cA}$ & $5 \mathrm{cA}$ & $5 \mathrm{dA}$ & $15 \mathrm{bA}$ & $17 \mathrm{bA}$ & $15 \mathrm{aA}$ & $9 \mathrm{cB}$ \\
\hline [DIU + HEX] & $7 \mathrm{cA}$ & $12 \mathrm{cA}$ & $8 \mathrm{cA}$ & $9 \mathrm{cA}$ & $14 \mathrm{bA}$ & $11 \mathrm{cA}$ & $14 \mathrm{aA}$ & $14 \mathrm{bA}$ \\
\hline MET & $7 \mathrm{cA}$ & $5 \mathrm{dA}$ & $0 \mathrm{dA}$ & $3 \mathrm{dA}$ & $11 \mathrm{bA}$ & $6 \mathrm{cB}$ & $1 \mathrm{cB}$ & $3 \mathrm{~dB}$ \\
\hline CLO & $22 \mathrm{aB}$ & $35 \mathrm{aA}$ & $30 \mathrm{aA}$ & $22 \mathrm{aB}$ & $24 \mathrm{aA}$ & $21 \mathrm{bA}$ & $17 \mathrm{aA}$ & $20 \mathrm{aA}$ \\
\hline $\mathrm{SAF}+\mathrm{AME}$ & $15 \mathrm{bA}$ & $16 \mathrm{cA}$ & $14 \mathrm{bA}$ & $12 \mathrm{cA}$ & $12 \mathrm{bA}$ & $16 \mathrm{bA}$ & $14 \mathrm{aA}$ & $17 \mathrm{aA}$ \\
\hline SAF + SUL & $19 \mathrm{aB}$ & $24 \mathrm{bA}$ & $16 \mathrm{bB}$ & $15 \mathrm{bB}$ & $19 \mathrm{aB}$ & $30 \mathrm{aA}$ & $17 \mathrm{aB}$ & $14 \mathrm{bB}$ \\
\hline $\mathrm{SAF}+\mathrm{TEB}$ & $15 \mathrm{bA}$ & $15 \mathrm{cA}$ & $12 \mathrm{bA}$ & $11 \mathrm{cA}$ & $11 \mathrm{bA}$ & $17 \mathrm{bA}$ & $15 \mathrm{aA}$ & $14 \mathrm{bA}$ \\
\hline $\mathrm{SAF}+[\mathrm{DIU}+\mathrm{HEX}]$ & $12 \mathrm{bA}$ & $16 \mathrm{cA}$ & $19 \mathrm{aA}$ & $17 \mathrm{bA}$ & $14 \mathrm{bA}$ & $20 \mathrm{bA}$ & $16 \mathrm{aA}$ & $21 \mathrm{aA}$ \\
\hline $\mathrm{SAF}+\mathrm{MET}$ & $14 \mathrm{bA}$ & $14 \mathrm{cA}$ & $12 \mathrm{bA}$ & $10 \mathrm{cA}$ & $14 \mathrm{bA}$ & $12 \mathrm{cA}$ & $11 \mathrm{aA}$ & $11 \mathrm{bA}$ \\
\hline $\mathrm{SAF}+\mathrm{CLO}$ & $15 \mathrm{bB}$ & $27 \mathrm{bA}$ & $24 \mathrm{aA}$ & $19 \mathrm{bB}$ & $21 \mathrm{aB}$ & $27 \mathrm{aA}$ & $24 \mathrm{aA}$ & $16 \mathrm{bB}$ \\
\hline Testemunha & $0 \mathrm{dA}$ & $0 \mathrm{dA}$ & $0 \mathrm{dA}$ & $0 \mathrm{dA}$ & $0 \mathrm{cA}$ & $0 \mathrm{dA}$ & $0 \mathrm{cA}$ & $0 \mathrm{dA}$ \\
\hline $\mathrm{CV}(\%)$ & & \multicolumn{4}{|c|}{31} \\
\hline
\end{tabular}

SAF = saflufenacil; AME = ametryn; SUL = sulfentrazone; TEB = tebuthiuron; HEX = hexazinone; DIU = diuron; MET = metribuzin; $\mathrm{CLO}=$ clomazone. Médias seguidas por letras minúsculas iguais na coluna e maiúsculas na linha não diferem entre si pelo teste de Scott-Knott ao nível de 5\% de probabilidade.

Tabela 8. Estatura (cm), área foliar $\left(\mathrm{cm}^{2}\right)$ e biomassa $(\mathrm{g})$ das mudas pré-brotadas, na variedade RB 96 6928, submetidas a diferentes herbicidas aos 3 e 10 DAP.

\begin{tabular}{|c|c|c|c|c|c|c|}
\hline \multirow{2}{*}{ Tratamentos } & \multicolumn{3}{|c|}{3 DAP } & \multicolumn{3}{|c|}{10 DAP } \\
\hline & Estatura & Área foliar & Biomassa & Estatura & Área foliar & Biomassa \\
\hline SAF & $5,75 \mathrm{c}$ & $53,60 \mathrm{~b}$ & $0,60 \mathrm{a}$ & $7,75 \mathrm{~b}$ & $50,87 \mathrm{~b}$ & $0,12 \mathrm{~b}$ \\
\hline AME & $5,25 \mathrm{c}$ & $62,32 \mathrm{~b}$ & $0,64 \mathrm{a}$ & $9,00 \mathrm{a}$ & $84,84 \mathrm{a}$ & $0,40 \mathrm{a}$ \\
\hline SUL & $5,25 \mathrm{c}$ & $95,70 \mathrm{a}$ & $0,54 \mathrm{a}$ & $9,05 \mathrm{a}$ & $61,47 \mathrm{a}$ & $0,20 \mathrm{~b}$ \\
\hline TEB & $5,75 \mathrm{c}$ & $82,13 \mathrm{a}$ & $0,52 \mathrm{a}$ & $8,00 \mathrm{a}$ & $76,15 \mathrm{a}$ & $0,47 \mathrm{a}$ \\
\hline$[\mathrm{DIU}+\mathrm{HEX}]$ & $6,50 \mathrm{~b}$ & $81,23 \mathrm{a}$ & $0,52 \mathrm{a}$ & $9,50 \mathrm{a}$ & $64,18 \mathrm{a}$ & $0,17 \mathrm{~b}$ \\
\hline MET & $5,25 \mathrm{c}$ & $78,50 \mathrm{a}$ & $0,57 \mathrm{a}$ & $7,25 \mathrm{~b}$ & $67,55 \mathrm{a}$ & $0,37 \mathrm{a}$ \\
\hline CLO & $4,50 \mathrm{c}$ & $55,30 \mathrm{~b}$ & $0,33 \mathrm{a}$ & $6,80 \mathrm{~b}$ & $51,87 \mathrm{~b}$ & $0,22 \mathrm{~b}$ \\
\hline $\mathrm{SAF}+\mathrm{AME}$ & $5,00 \mathrm{c}$ & 88,89 a & $0,45 \mathrm{a}$ & $6,80 \mathrm{~b}$ & $65,33 \mathrm{a}$ & $0,24 \mathrm{~b}$ \\
\hline $\mathrm{SAF}+\mathrm{SUL}$ & $4,75 \mathrm{c}$ & $56,27 \mathrm{~b}$ & $0,63 \mathrm{a}$ & $6,75 b$ & $41,74 \mathrm{~b}$ & $0,31 \mathrm{~b}$ \\
\hline $\mathrm{SAF}+\mathrm{TEB}$ & $4,50 \mathrm{c}$ & $53,53 \mathrm{~b}$ & $0,46 \mathrm{a}$ & $7,75 \mathrm{~b}$ & $52,32 \mathrm{~b}$ & $0,16 \mathrm{~b}$ \\
\hline $\mathrm{SAF}+[\mathrm{DIU}+\mathrm{HEX}]$ & $4,25 \mathrm{c}$ & $64,77 \mathrm{~b}$ & $0,40 \mathrm{a}$ & $6,05 \mathrm{~b}$ & $45,89 \mathrm{~b}$ & $0,19 \mathrm{~b}$ \\
\hline $\mathrm{SAF}+\mathrm{MET}$ & $5,75 \mathrm{c}$ & $78,17 \mathrm{a}$ & $0,59 \mathrm{a}$ & $9,00 \mathrm{a}$ & $79,75 \mathrm{a}$ & $0,63 \mathrm{a}$ \\
\hline $\mathrm{SAF}+\mathrm{CLO}$ & $4,75 \mathrm{c}$ & $49,57 \mathrm{~b}$ & $0,43 \mathrm{a}$ & $8,75 \mathrm{a}$ & $71,24 \mathrm{a}$ & $0,20 \mathrm{~b}$ \\
\hline Testemunha & $8,15 \mathrm{a}$ & $68,81 \mathrm{~b}$ & $0,56 \mathrm{a}$ & $8,15 \mathrm{a}$ & $68,81 \mathrm{a}$ & $0,56 \mathrm{a}$ \\
\hline CV $(\%)$ & 19,23 & 18,58 & 46,92 & 16,42 & 19,16 & 76,17 \\
\hline
\end{tabular}

SAF = saflufenacil; AME = ametryn; SUL = sulfentrazone; TEB = tebuthiuron; HEX = hexazinone; DIU = diuron; MET = metribuzin; $\mathrm{CLO}=$ clomazone. Médias seguidas por letras minúsculas iguais na coluna e maiúsculas na linha não diferem entre si pelo teste de Scott-Knott ao nível de 5\% de probabilidade.

$\mathrm{Na}$ aplicação realizada aos 10 DAP houve diferenças nos parâmetros biométricos das plantas para alguns tratamentos. Apenas o saflufenacil + metribuzin, metribuzin, tebuthiuron $\mathrm{e}$ ametryn não diferiram da testemunha quanto à biomassa seca, sendo que os demais tratamentos apresentaram redução na biomassa.

Os resultados mostram que as variedades diferem em relação à tolerância aos herbicidas e este fato pode ser atribuído às características genéticas específicas de cada material (Tabela 8). Além disto, o padrão de 
crescimento e recuperação da intoxicação pouco depende da intensidade inicial de intoxicação das cultivares de cana-de-açúcar, e, portanto, apesar de alta toxicidade início do período de avaliação, a cultura poderia se recuperar e produzir colmos sadios (Martins et al., 2010).

Trabalhando com a mesma variedade, em aplicação pré-plantio de diclosulam, Dias (2014) observou que as mudas pré-brotadas de RB 96 6928, embora apresentassem fitotoxicidade menor que $30 \%$, tiveram a biomassa seca de parte aérea reduzida, o que corrobora os resultados do presente trabalho.

A variedade RB 975201 apresentou maior suscetibilidade a alguns herbicidas em relação às demais variedades. Na aplicação realizada aos 3 DAP, os herbicidas que apresentaram maior fitotoxicidade nas mudas aos 60 DAA foram o clomazone (77\%), saflufenacil + ametryn (61\%) e diuron + hexazinone (64\%), inclusive com aumento de fitotoxicidade destes tratamentos ao longo das avaliações. Esses resultados mostram a inviabilização da aplicação destes herbicidas em curto período de tempo após o plantio das mudas desta variedade no campo (Tabela 9). Na aplicação aos 10 DAP, observou-se maior tolerância desta variedade aos herbicidas, sendo que aos 60 DAA os tratamentos que provocaram maior fitotoxicidade foram: saflufenacil + metribuzin com $34 \%$ de fitotoxicidade, seguido por saflufenacil + diuron + hexazinone com $27 \%$, clomazone com 25\%, saflufenacil + clomazone com $21 \%$ e hexazinone + diuron com $20 \%$ de fitotoxicidade (Tabela 9 ).

Tabela 9. Porcentagem de fitotoxicidade dos herbicidas aplicados nas mudas pré-brotadas, na variedade RB 97 5201, após 3 e 10 DAP.

\begin{tabular}{|c|c|c|c|c|c|c|c|c|}
\hline \multirow{2}{*}{ Tratamentos } & \multicolumn{4}{|c|}{$3 \mathrm{DAP}$} & \multicolumn{4}{|c|}{$10 \mathrm{DAP}$} \\
\hline & $7 \mathrm{DAA}$ & $15 \mathrm{DAA}$ & $30 \mathrm{DAA}$ & $60 \mathrm{DAA}$ & $7 \mathrm{DAA}$ & $15 \mathrm{DAA}$ & $30 \mathrm{DAA}$ & $60 \mathrm{DAA}$ \\
\hline $\mathrm{SAF}$ & $12 \mathrm{aA}$ & $20 \mathrm{bA}$ & $20 \mathrm{bA}$ & $25 \mathrm{bA}$ & $20 \mathrm{aA}$ & $14 \mathrm{aA}$ & $11 \mathrm{cA}$ & $12 \mathrm{bA}$ \\
\hline AME & $10 \mathrm{aA}$ & $14 \mathrm{bA}$ & $10 \mathrm{bA}$ & $9 \mathrm{bB}$ & $6 \mathrm{bA}$ & $6 \mathrm{bA}$ & $11 \mathrm{cA}$ & $12 \mathrm{bA}$ \\
\hline SUL & $12 \mathrm{aA}$ & $15 \mathrm{bA}$ & $9 \mathrm{bA}$ & $11 \mathrm{bA}$ & $29 \mathrm{aA}$ & $27 \mathrm{aA}$ & $17 \mathrm{cB}$ & $15 \mathrm{bB}$ \\
\hline TEB & $11 \mathrm{aA}$ & $14 \mathrm{bA}$ & $12 \mathrm{bA}$ & $12 \mathrm{bA}$ & $10 \mathrm{bA}$ & $10 \mathrm{bA}$ & $5 \mathrm{dA}$ & $10 \mathrm{bA}$ \\
\hline [DIU + HEX] & $14 \mathrm{aB}$ & $39 \mathrm{aA}$ & $46 \mathrm{aA}$ & $64 \mathrm{aA}$ & $12 \mathrm{bA}$ & $9 \mathrm{bA}$ & $16 \mathrm{cA}$ & $20 \mathrm{aA}$ \\
\hline MET & $3 \mathrm{aA}$ & $3 \mathrm{bA}$ & $0 \mathrm{bA}$ & $4 \mathrm{bA}$ & $5 \mathrm{bA}$ & $3 \mathrm{bA}$ & $0 \mathrm{dA}$ & $5 \mathrm{bA}$ \\
\hline CLO & $12 \mathrm{aB}$ & $26 \mathrm{aB}$ & $27 \mathrm{aB}$ & $77 \mathrm{aA}$ & $20 \mathrm{aA}$ & $17 \mathrm{aA}$ & $24 \mathrm{bA}$ & $25 \mathrm{aA}$ \\
\hline $\mathrm{SAF}+\mathrm{AME}$ & $14 \mathrm{aB}$ & $31 \mathrm{aB}$ & $36 \mathrm{aB}$ & $61 \mathrm{aA}$ & $20 \mathrm{aA}$ & $19 \mathrm{aA}$ & $10 \mathrm{cA}$ & $10 \mathrm{bA}$ \\
\hline SAF + SUL & $21 \mathrm{aA}$ & $32 \mathrm{aA}$ & $30 \mathrm{aA}$ & $27 \mathrm{bA}$ & $24 \mathrm{aA}$ & $19 \mathrm{aA}$ & $15 \mathrm{cA}$ & $10 \mathrm{bA}$ \\
\hline $\mathrm{SAF}+\mathrm{TEB}$ & $14 \mathrm{bA}$ & $16 \mathrm{bA}$ & $11 \mathrm{bA}$ & $10 \mathrm{bA}$ & $21 \mathrm{aA}$ & $16 \mathrm{aA}$ & $11 \mathrm{cA}$ & $14 \mathrm{bA}$ \\
\hline $\mathrm{SAF}+[\mathrm{DIU}+\mathrm{HEX}]$ & $11 \mathrm{aA}$ & $17 \mathrm{bA}$ & $15 \mathrm{bA}$ & $15 \mathrm{bA}$ & $35 \mathrm{aA}$ & $19 \mathrm{aB}$ & $45 \mathrm{aA}$ & $27 \mathrm{aB}$ \\
\hline $\mathrm{SAF}+\mathrm{MET}$ & $14 \mathrm{aA}$ & $15 \mathrm{bA}$ & $11 \mathrm{bA}$ & $5 \mathrm{bA}$ & $15 \mathrm{bB}$ & $15 \mathrm{aB}$ & $34 \mathrm{bA}$ & $34 \mathrm{aA}$ \\
\hline $\mathrm{SAF}+\mathrm{CLO}$ & $22 \mathrm{aA}$ & $34 \mathrm{aA}$ & $27 \mathrm{aA}$ & $24 \mathrm{bA}$ & $30 \mathrm{aA}$ & $22 \mathrm{aA}$ & $26 \mathrm{bA}$ & $21 \mathrm{aA}$ \\
\hline Testemunha & $0 \mathrm{aA}$ & $0 \mathrm{bA}$ & $0 \mathrm{bA}$ & $0 \mathrm{bA}$ & $0 \mathrm{bA}$ & $0 \mathrm{bA}$ & $0 \mathrm{dA}$ & $0 \mathrm{bA}$ \\
\hline $\mathrm{CV}(\%)$ & \multicolumn{4}{|c|}{85} & \multicolumn{4}{|c|}{58} \\
\hline
\end{tabular}

SAF = saflufenacil; AME = ametryn; SUL = sulfentrazone; TEB = tebuthiuron; HEX = hexazinone; DIU = diuron; MET = metribuzin; $\mathrm{CLO}=$ clomazone. Médias seguidas por letras minúsculas iguais na coluna e maiúsculas na linha não diferem entre si pelo teste de Scott-Knott ao nível de 5\% de probabilidade.

A aplicação dos herbicidas aos 3 DAP da variedade RB 975201 resultou em efeito negativo na estatura, biomassa e área foliar, com destaque para os tratamentos diuron + hexazinone, clomazone, saflufenacil + ametryn, saflufenacil + (diuron + hexazinone) $\mathrm{e}$ saflufenacil + sulfentrazone. Aos 10 DAP os herbicidas sulfentrazone, diuron + hexazinone, clomazone e as associações de saflufenacil com ametryn, tebuthiuron, e diuron + hexazinone reduziram as variáveis estatura e biomassa (Tabela 10).

O estádio fenológico da cana-de-açúcar influencia a tolerância da planta aos herbicidas aplicados, sendo importante a avaliação do 
estádio fenológico da cultura, antes de se realizar as aplicações com herbicidas.

A cana-de-açúcar, em condição de cana planta, mostra-se tolerante a herbicidas residuais que são absorvidos pela raiz, quando aplicados no estádio de esporão da cultura, pois nesta fase não existe uma ligação direta dos vasos do xilema da raiz com o caule da brotação.

Tabela 10. Estatura $(\mathrm{cm})$, área foliar $\left(\mathrm{cm}^{2}\right)$ e biomassa $(\mathrm{g})$ das mudas pré-brotadas, na variedade RB 97 5201, submetidas a diferentes herbicidas aos 3 e 10 DAP.

\begin{tabular}{lcccccc}
\hline \multirow{2}{*}{ Tratamentos } & \multicolumn{3}{c}{3 DAP } & \multicolumn{3}{c}{ 10 DAP } \\
\cline { 2 - 7 } & Estatura & Área foliar & Biomassa & Estatura & Área foliar & Biomassa \\
\hline SAF & $7,50 \mathrm{a}$ & $55,96 \mathrm{a}$ & $0,67 \mathrm{a}$ & $10,00 \mathrm{a}$ & $78,42 \mathrm{a}$ & $0,93 \mathrm{a}$ \\
AME & $5,50 \mathrm{~b}$ & $57,73 \mathrm{a}$ & $0,73 \mathrm{a}$ & $7,72 \mathrm{~b}$ & $83,31 \mathrm{a}$ & $0,34 \mathrm{~b}$ \\
SUL & $7,25 \mathrm{a}$ & $73,68 \mathrm{a}$ & $0,65 \mathrm{a}$ & $7,57 \mathrm{~b}$ & $57,22 \mathrm{~b}$ & $0,20 \mathrm{~b}$ \\
TEB & $6,00 \mathrm{~b}$ & $68,28 \mathrm{a}$ & $0,62 \mathrm{a}$ & $10,25 \mathrm{a}$ & $91,27 \mathrm{a}$ & $0,56 \mathrm{a}$ \\
[DIU + HEX] & $3,50 \mathrm{~b}$ & $29,81 \mathrm{~b}$ & $0,11 \mathrm{~b}$ & $7,50 \mathrm{~b}$ & $43,95 \mathrm{~b}$ & $0,25 \mathrm{~b}$ \\
MET & $6,50 \mathrm{~b}$ & $62,66 \mathrm{a}$ & $0,66 \mathrm{a}$ & $9,50 \mathrm{a}$ & $73,78 \mathrm{a}$ & $0,74 \mathrm{a}$ \\
CLO & $4,75 \mathrm{~b}$ & $31,01 \mathrm{~b}$ & $0,19 \mathrm{~b}$ & $7,25 \mathrm{~b}$ & $40,29 \mathrm{~b}$ & $0,26 \mathrm{~b}$ \\
SAF + AME & $5,00 \mathrm{~b}$ & $30,43 \mathrm{~b}$ & $0,21 \mathrm{~b}$ & $8,25 \mathrm{~b}$ & $52,95 \mathrm{~b}$ & $0,41 \mathrm{~b}$ \\
SAF + SUL & $5,50 \mathrm{~b}$ & $56,14 \mathrm{a}$ & $0,39 \mathrm{~b}$ & $10,00 \mathrm{a}$ & $94,76 \mathrm{a}$ & $0,67 \mathrm{a}$ \\
SAF + TEB & $5,50 \mathrm{~b}$ & $47,27 \mathrm{~b}$ & $0,51 \mathrm{a}$ & $7,62 \mathrm{~b}$ & $59,78 \mathrm{~b}$ & $0,22 \mathrm{~b}$ \\
SAF + [DIU + HEX] & $5,25 \mathrm{~b}$ & $47,70 \mathrm{~b}$ & $0,31 \mathrm{~b}$ & $5,50 \mathrm{~b}$ & $32,86 \mathrm{~b}$ & $0,19 \mathrm{~b}$ \\
SAF + MET & $5,75 \mathrm{~b}$ & $75,37 \mathrm{a}$ & $0,46 \mathrm{a}$ & $8,52 \mathrm{a}$ & $54,41 \mathrm{~b}$ & $0,41 \mathrm{~b}$ \\
SAF + CLO & $5,75 \mathrm{~b}$ & $61,87 \mathrm{a}$ & $0,47 \mathrm{a}$ & $9,25 \mathrm{a}$ & $76,56 \mathrm{a}$ & $0,34 \mathrm{~b}$ \\
Testemunha & $9,10 \mathrm{a}$ & $56,31 \mathrm{a}$ & $0,79 \mathrm{a}$ & $9,10 \mathrm{a}$ & $56,31 \mathrm{~b}$ & $0,79 \mathrm{a}$ \\
\hline CV $(\%)$ & 25,01 & 33,71 & 51,19 & 15,41 & 28,20 & 57,12 \\
\hline SAF = & & &
\end{tabular}

SAF = saflufenacil; AME = ametryn; SUL = sulfentrazone; TEB = tebuthiuron; HEX = hexazinone; DIU = diuron; MET = metribuzin; $\mathrm{CLO}=$ clomazone. Médias seguidas por letras minúsculas iguais na coluna e maiúsculas na linha não diferem entre si pelo teste de Scott-Knott ao nível de 5\% de probabilidade.

O estádio fenológico 2, de duas a três folhas, a cana-de-açúcar é muito sensível a herbicidas foliares, pois, as folhas ainda apresentam uma cutícula mais fina, o que implica em menor resistência à penetração dos herbicidas. Como o crescimento inicial da canade-açúcar depende do colmo primário que é formado neste estádio, qualquer injúria severa pode resultar em perda de produtividade final da cultura (Arantes et al., 2013). O estádio fenológico 2, é o estádio em que se apresentam as mudas pré-brotadas de cana-de-açúcar no momento do plantio. Assim podendo ser mais sensíveis aos herbicidas aplicados e ocasionando perda de produtividade.

\section{Conclusões}

As variedades RB $867515, \mathrm{RB} 85$ 5156, RB 966928 e RB 975201 tiveram tolerância diferencial aos herbicidas avaliados e as épocas de aplicação.
A variedade RB 867515 teve maior tolerância aos herbicidas nas duas épocas avaliadas. Os tratamentos que provocaram maior fitotoxicidade foram saflufenacil + clomazone, saflufenacil + ametryn e tebuthiuron aos 3 DAP e saflufenacil + clomazone aos 10 DAP.

As plantas da variedade RB 855156 apresentaram maior fitotoxicidade quando tratadas com clomazone aos 3 e 10 DAP. Com relação à variedade RB 96 6928, na aplicação aos 3 DAP, o tratamento com clomazone provocou a maior fitotoxicidade e aos 10 DAP destacaram-se os tratamentos clomazone e saflufenacil + diuron + hexazinone.

A variedade RB 975201 apresentou os maiores valores de fitotoxicidade, destacandose na aplicação realizada aos 3 DAP, os tratamentos hexazinone + diuron, saflufenacil + ametryn e na aplicação aos 10 DAP os tratamentos hexazinone + diuron, clomazone, 
(saflufenacil + diuron + hexazione) $\mathrm{e}$ saflufenacil + clomazone.

Para as variedades RB 85 5156, RB 96 6928 e RB 975201 a época mais adequada de aplicação varia de acordo com o herbicida utilizado.

\section{Referências}

Arantes, M.T.; Rhein, A.F.L.; Pincelli R.P.; Silva, M.A. Bioscience Journal, v.29, n.5, p.1206-1214, 2013.

Asociation Latinoamericana De Malezas. Recomendaciones sobre unificación de los sistemas de evaluación em ensayos de control de malezas. ALAM, v.1, n.1, p.35-38, 1974.

Barela, J.F.; Christoffoleti, P.J. Seletividade de herbicidas aplicados em pré-emergência da cultura da cana-de-açúcar (RB 867515) tratada com nematicidas. Planta Daninha, v.24, n.2, p.371-378, 2006.

Rocha Neto, A. R.; Azania, C. A. M.; Borges, I.V.; Vitorino, R.; Marchine, N. M.; Azania, A.P.M. Tolerância de mudas pré-brotadas de cana-de-açúcar a herbicidas aplicados em pósemergência. In: IX Workshop de Agroenergia, 9., 2015, Ribeirão Preto. Anais... Ribeirão Preto: APTA, 2015. p.1-6.

Costa, S.I.A.; Martins, D.; Cardoso, L.A.; Rodrigues, A.C.P.; Vitorino, H.S.; Marques, R.P. Seletividade do herbicida saflufenacil aplicado em pós-emergência em dez variedades de cana-de-açúcar na condição de soca. Arquivo do Instituto Biológico, v.79, n.1, p.113-120, 2012.

Dias, J.L.C.S. Seletividade de herbicidas em mudas pré- brotadas de cana-de-açúcar. 2014. 73f. Dissertação (Mestrado em Fitotecnia) - Universidade Estadual Paulista, Jaboticabal, 2014.

Ferreira, E.A.; Santos, J.B.; Silva, A.A.; Ventrella, M.C.; Barbosa, M.H.P.; Procópio, S.O. et al. Sensibilidade de cultivares de canade-açúcar à mistura trifloxysulfuron-sodium + ametrina. Planta Daninha, v.23, n.1, p.93-99, 2005.

Ferreira, E. A.; Silva, A.F.; Silva, A.A.; Silva, D.V.; Galon, L.L.; França, A.C. et al. Toxidade de herbicidas a genótipos de cana-de-açúcar. Revista Trópica: Ciências Agrárias e Biológicas, v.6, n.1, p.84-92, 2012.

Ferreira, R.R.; Oliveira, F.T.R.; Delite, F.S.; Azevedo, R.A.; Nicolai, M.; Carvalho, S.J.P. et al. Tolerância diferencial de variedades de canade-açúcar a estresse por herbicidas. Bragantia, v.69, n.2, p.395-404, 2010.

Inoue, M.H.; Santin, A.j.; Dallacort, R.; Possamai, A.S.; Santana, D.C. Performance de associações de herbicidas em cana-de-açúcar (Saccharum officinarum). Revista Brasileira de Herbicidas, v.6, n.2, p.32-41, 2007.

Landell, M.G.A.; Campanha, M.P.; Figueiredo, P.; Xavier, M.A.; Anjos, I.A.; Dinardo-Miranda, L.L. et al. Sistema de Multiplicação de cana-deaçúcar com uso de mudas pré-brotadas (MPB) oriundas de gemas individualizadas. Campinas: Documentos IAC - Boletim Técnico, v.109, n.2, 16 p, 2013.

Martins, D.; Costa, N.V.; Cardoso, L.A.; Rodrigues, A.C.P.; Silva, J.I.C. Seletividade de herbicidas em variedades de cana-de-açúcar. Planta Daninha, v.28, n.esp., p.1125-1134, 2010.

Martins, D.; Velini, E.D.; Negrisoli, E.; Marchi, S.R.; Silva, J. R. V. Seletividade do herbicida diclosulam, aplicado em pré e pós emergência em diversas cultivares de cana-de-açúcar. Revista Brasileira de Herbicidas, v.4, n.2, p.19, 2005.

Montório, G.A.; Constantin, J.; Velini, E.D.; Montório, T. Seletividade de herbicidas sobre as características de produção da cana-de-açúcar utilizando-se duas testemunhas. Revista Brasileira de Herbicidas, v. 4, n.1, p.146-156, 2005.

Pedrinho Junior, A.F.F.; Martini, G.; Durigan, J.C. Controle de tiririca (Cyperus rotundus) na 
cultura da cana-de-açúcar, com o herbicida imazapic isolado ou em mistura com pendimenthalin. Revista Brasileira de Herbicidas, v.2, n.1, p.23-29, 2001.

Torres, L.G.; Ferreira, E.A.; Rocha, P.R.R.; Faria, A.T.; Gonçalves, V.A.; Galon, L. et al. Alterações nas características fisiológicas de cultivares de cana-de-açúcar submetida à aplicação de herbicidas. Planta Daninha, v.30, n.3, p.581-587, 2012.

Victoria Filho, R.; Christoffoleti, P.J. Manejo de plantas daninhas e produtividade da cana. Visão Agrícola, v.1, n.1, p.32-37, 2004. 